\title{
Caregivers' Willingness to Vaccinate Their Children Against Childhood Diseases and Human Papillomavirus: a Cross- sectional Study on Vaccine Hesitancy in Malawi
}

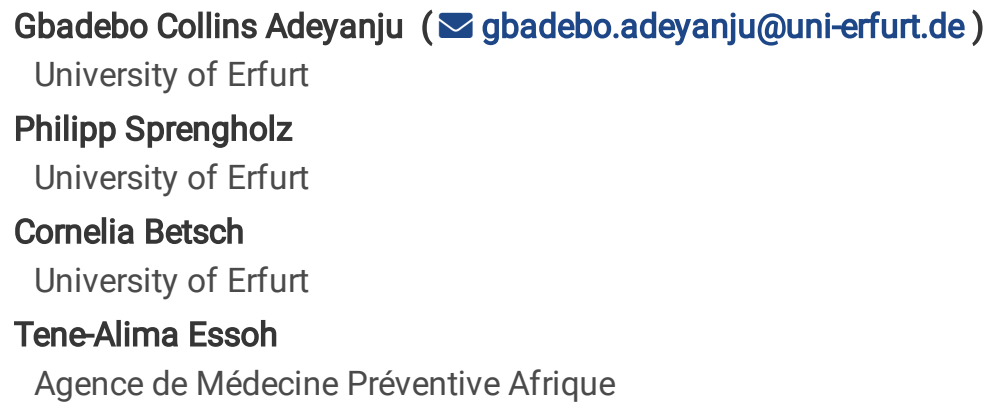

\section{Research Article}

Keywords: Immunization, Vaccine hesitancy, Vaccine demand, Vaccination, Malawi, Drivers, Caregivers, Childhood diseases, Human papillomavirus (HPV), Adolescent

Posted Date: June 21st, 2021

DOI: https://doi.org/10.21203/rs.3.rs-618575/v1

License: @ (i) This work is licensed under a Creative Commons Attribution 4.0 International License. Read Full License 


\section{Abstract}

Background: Vaccines are among the most effective and cost-efficient public health interventions for promoting child health. However, uptake is considerably affected by vaccine hesitancy. Malawi has experienced a decline in second vaccine doses and has the highest cervical cancer incidence and mortality rate in Sub-Saharan Africa. Understanding vaccine hesitancy is especially important when new vaccines are introduced. This study explores factors contributing to vaccine hesitancy for routine childhood immunization and the human papillomavirus vaccine in Malawi.

Methods: The study used a cross-sectional survey design to target caregivers of children under five years of age and adolescent girls. The sample population was derived using three inclusion criteria: one district with low vaccine uptake (Dowa), one district with high vaccine uptake (Salima), and one district where human papillomavirus vaccine was piloted earlier (Zomba). A convenience sample of one primary and one secondary health facility was selected within each district, and participants were systematically included $(N=600)$. Multiple regression analyses were performed to explore vaccination intention predictors.

Results: Confidence in vaccine safety was the strongest predictor of routine childhood immunization, followed by constraints due to everyday stress. The participants had lower confidence in vaccine safety and efficacy when they believed rumors and misinformation and were unemployed. Confidence was higher for those who had more trust in healthcare workers. Age, gender, religion, education, employment, belief in rumors, and trust in healthcare workers were considered predictors of vaccination intention. A husband's positive attitude (approval) increased childhood vaccination intention. For human papillomavirus, vaccination intentions were higher for those with lower education, more trust in healthcare workers, lower complacency, and a lower tendency toward calculating the benefits and costs of vaccination. Knowledge of human papillomavirus did not increase vaccination intention, but a husband's approval did. Being a younger adult and being unemployed increased belief in rumors, while trust in healthcare workers reduced it.

Conclusions: This study provides insights into the drivers of vaccine hesitancy across different contexts in Malawi. However, further studies are necessary to understand low risk perception among elderly people and the declining trend in second vaccine doses.

\section{Background}

Vaccination is regarded as one of the most effective and cost-effective public health innovations for promoting child health because of its direct health benefits and positive externalities [1]. It can prevent morbidity and mortality from vaccine-preventable diseases and contribute to national disease elimination and eradication efforts [2]. The World Health Organization (WHO) and the United Nations Children's Emergency Fund (UNICEF) estimate that vaccination prevents about two to three million deaths in children annually [3]. In addition, remarkable progress has been made toward global polio eradication due to global vaccination programs and efforts $[3,4]$. To ensure sustainable and equitable access to vaccines, countries coordinate immunization-related activities within global and national immunization programs. One indicator to measure programs' performance is the coverage rate with the third dose of the diphtheria-tetanus-pertussis-containing vaccine (DTP3), which is expected to be $90 \%$ at the national level and $80 \%$ at the district level $[5,6]$. However, in 2018, the global average for DTP3 coverage was about $86 \%$ [7].

Between 2000 and 2019, over 822 million children were immunized worldwide, 1.1 billion vaccinations were supported via multiple campaigns, an estimated 14 million deaths were averted, and 150 billion USD economic benefits were generated due to immunization [8]. An additional 300 million children were to be immunized against potentially fatal diseases by the end of 2020 , saving between five and six million lives, preventing 250 million disability-adjusted life years, and reducing under-five mortality by $10 \%[9,10]$.

Despite this considerable progress, one in five children globally remains unvaccinated or partially vaccinated, which contributes to about 1.5 million deaths from vaccine-preventable diseases annually [11-13]. In the WHO African region, DTP3 coverage has stagnated at $76 \%$ [14], and the region contribute the highest proportion of under-vaccinated and consequent child deaths from vaccine-preventable diseases globally [15-17]. Of the 10 countries that account for 11.7 (60\%) of the 19.7 million non- or undervaccinated children globally, $40 \%$ are in Sub-Saharan Africa (SSA), including Nigeria, Ethiopia, Democratic Republic of Congo, and Angola [18]. Average coverage rates in the SSA region remain sub-optimal, stagnating over the past five years at $72 \%[19,20]$. 
Despite the promise of vaccines recent data have shown a different and negative, behavior to vaccination uptake, which can be regarded as vaccine hesitancy $[21,22]$.

In Malawi, the percentage of fully immunized children aged 12-23 months has been declining since 1992, when coverage peaked at $82 \%$ [23]. This declined to $64 \%$ in 2004 , then rose to $81 \%$ and $99 \%$ in 2010 and 2012, respectively [23]. In 2018, the current national average vaccination coverage rates for three doses of DTP3 in Malawi declined again to 92\% [24].

A study on vaccination coverage and timeliness with valid doses in Malawi showed that, while the availability of vaccination cards (evidence of inoculation) in the Dowa and Ntchisi districts was as high as $94 \%$ and vaccination coverage by card and mothers' history was also as high as $93 \%$ for all antigens, the percentages of valid doses completed by children was $60 \%$ in Dowa District and as low as $49 \%$ in Ntchisi District [25]. The assessment showed that many children in the two districts often had an incomplete number of doses.

\section{Cervical cancer burden and HPV vaccination}

Cervical cancer will kill more than 443,000 women per year worldwide by 2030 , and nearly $90 \%$ of the deaths will be in SSA [26, 27]. The burden of this disease is most severe in low- and middle-income countries. The top 20 countries with the highest burden of cervical cancer cases globally in 2018 were all in SSA, except for Bolivia [28, 29]. Within SSA, East Africa has the highest incidence rate, where Malawi is among not just the global leaders but also top in the former [24, 29]. Malawi has the second highest burden of cervical cancer globally and the highest in the SSA region [27]. It is the most common cancer in women in the country, accounting for $45.4 \%$ of all female cancer incidence $[29,30]$. Of all diagnosed cancers among women in Malawi, $80 \%$ will die prematurely [31, 32]. Overall, about 5 million Malawian women aged 15-44 are most at risk of developing cervical cancer [33, 34].

Cervical cancer is caused by the human papillomavirus (HPV), a double-stranded DNA virus [35]. It is one of the most prevalent sexually transmitted diseases, with over half of sexually active individuals contracting HPV during their life [36]. Despite the existence of over 200 HPV types, most HPV-related pathologies are due to infection with HPV types 16, 18, 6, and 11[37, 38].

The HPV vaccine was introduced into the routine immunization (RI) program in Malawi to reduce the high rate of cervical cancer deaths among women [39]. Through vaccination, cervical cancer can be prevented by vaccinating adolescent girls before they become sexually active, helping to reduce the spread of the virus and consequently lower cervical cancer mortality. Prior to its introduction into the RI program in Malawi in 2019, the pilot demonstration (2013-2016) successfully vaccinated 26,766 in-school girls and aimed to vaccinate 1.5 million adolescent girls ages $9-14[40,41]$. The pilot demonstration revealed a decline between the first and the second dose in the Rumphi $(98-88 \%)$ and Zomba districts $(89-76 \%)[42,43]$. Despite the possibility of safe protection against cervical cancer and vaccine availability, uptake remained low due to several factors including vaccine hesitancy.

\section{Vaccine hesitancy}

Although many factors may be responsible for low childhood and HPV vaccine coverage, vaccine hesitancy is recognized as an important contributor [22,44], hence the underlining motive of this study. The Strategic Advisory Group of Experts (SAGE) on Immunization defines vaccine hesitancy as a "delay in acceptance or refusal of vaccines despite availability of vaccination services" $[45,46]$. This definition suggests that vaccine hesitancy is a demand-side problem that influences vaccination uptake because of several complex factors, including perception about vaccines, fear of adverse events, religious values, and a general lack of trust in healthcare professionals or the healthcare system [47].

Vaccine hesitancy ranges from delay in acceptance of vaccines to complete refusal. It is driven by factors such as confidence (level of trust in vaccine or provider), complacency (not perceiving a need for vaccine or not valuing the vaccine), and convenience (access) [47]. It is also context-specific, as it depends on time, place, the specific vaccine, and the societal context. Therefore, this study will examine context- and vaccine-specific determinants of vaccine hesitancy to inform context-specific strategies, especially for new and underutilized vaccines such as the HPV vaccine.

Even in SSA, where vaccination has been the hallmark of public health intervention for development in the last 40 years, vaccine hesitancy is causing vaccination uptake to slow down, stagnate, or even decrease [45, 47-51]. In Malawi, the WHO/UNICEF Joint Reporting Form for 2018 named religious factors, perception, and lack of awareness as reasons for vaccine hesitancy [52]. 
However, the reporting was not grounded in evidence; instead, it relied on the opinions of field health officers [53]. The dearth of empirical evidence for understanding vaccine hesitancy has hindered robust and responsive intervention and therefore justifies this study.

A few studies have shown that caregivers who are hesitant about vaccination are more likely to attend vaccination appointments late [54]. Also, a study has shown an association between hesitancy and missed opportunities for vaccination in Malawi [55]. In Malawi, $66 \%$ of Malawian children eligible for vaccination did not receive at least one vaccination despite availability; in addition, $92 \%$ of people attending health facilities for non-vaccination visits and who were eligible for vaccination had at least one missed opportunity, and $57 \%$ have missed more [56]. Caregivers of adolescent girls who refused the HPV vaccination for their daughters or who did not complete doses during the HPV demonstration project in the Rumphi and Zomba districts of Malawi named reasons such as inconvenient location and time, belief that the vaccine portends danger to the girls, and the vaccination site being unclean and not safe [40]. Education about cervical cancer, inadequate information about vaccination opportunities, fears of side effects, and a general distrust toward new vaccines were some of the identified factors driving vaccine hesitancy in the early introduction of the HPV vaccine to the country $[57,58]$. Generally, because of the target population (adolescent girls) and amplified by rumors, mistrust of HPV vaccination seems widespread in low-income settings [59-61].

Currently, vaccine hesitancy is galvanizing unprecedented scholarly focus globally in view of the COVID-19 pandemic; however, at the same time, there is a vacuum of knowledge, especially in the Africa region. This limits the extent of evidence-based intervention in the region. No scientific model has yet been explored in Malawi that measures vaccine hesitancy and compares the relative impact of these influencing factors. Hence, this study's goal was to systematically explore factors that influence vaccine hesitancy in Malawi among caregivers of children and adolescent girls who are eligible for RI and HPV vaccination, respectively. Also, to assess the depth of vaccination knowledge among caregivers in Malawi. Multi-dimensional tools and ways to measure vaccine hesitancy exist, but little is known about their validity in non-Western settings such as SSA [51]. Other factors may be relevant in SSA, but no tool currently exists to assess and extend existing measures. Therefore, this study also assessed use of an expanded $5 \mathrm{C}$ psychological antecedents model to understand vaccine hesitancy drivers in Malawi.

\section{$5 \mathrm{C}+$ model for measuring vaccine hesitancy}

According to Betsch et al., there are five psychological antecedents of vaccination behavior represented in the $5 \mathrm{C}$ model that measures vaccines hesitancy: confidence, complacency, constraints, calculation, and collective responsibility [49].

"Confidence is trust in the effectiveness and safety of vaccines. Complacency exists where the perceived risks of vaccinepreventable diseases are low, and vaccination is not deemed a necessary preventive action. Constraints are an issue when physical availability, affordability, and willingness-to-pay, geographical accessibility, ability to understand (language and health literacy), and appeal of immunization service affect uptake. Calculation refers to individuals' engagement in extensive information searching and should therefore be related to perceived vaccination and disease risks. Collective responsibility is the willingness to protect others by one's own vaccination by means of herd immunity [49].

Religion, rumors, and masculinity were added to the set of items, referring to the augmented scale as " $5 \mathrm{C}+$." Religion has been found to be an important factor affecting people's attitudes toward vaccine demand [62-64]. Religious reasons for declining immunization reflect the role of beliefs among faith communities $[49,62,65]$. Masculinity is used here to connote a husband/father's role in the household's decision to vaccinate a child. A husband's approval (attitude) for the child to be vaccinated plays an important role in vaccination acceptance and refusal. Caregivers who solely depend on their husband's approval are prone to vaccinate less if the husband does not approve [66]. Finally, rumor/misinformation affects perceptions and everyday life, amplified in the age of social media, and this has been found to have some impact on vaccination demand in previous studies $[67,68]$. In addition, since knowledge is associated with HPV vaccination behavior, this variable was considered important in the assessment of vaccine hesitancy drivers in Malawi.

\section{Methods}

\section{Design and setting}


The study used a cross-sectional study design and was initially planned to be conducted in Rumphi, Dowa, Zomba and Nsanje districts. However, due to the SARS-CoV-2 pandemic situation in Malawi in April 2020, data were collected in the Salima, Dowa, and Zomba districts, which were selected based on the following criteria: one district with high vaccine coverage (Salima), one district with low vaccine coverage (Dowa), and one district where the HPV vaccination had been piloted (Zomba).

\section{Study population and sample size}

The study population included caregivers of children under five years of age and caregivers of adolescent girls whose children were eligible for HPV vaccination at local health centres. A total sample of 800 was planned ( $n=200$ per district), but a reduced sample of 600 participants ( $n=200$ per district) was enrolled due to the pandemic situation. The fourth district (Nsanje) was canceled due to travel restrictions.

\section{Sampling and data collection}

Within each stratum (district), a convenience sample of one primary and one secondary health facility was selected. For each sample, a systematic sampling technique was used to select study participants [69]: every third caregiver who visited the facility was included until sample saturation. Every caregiver who met the eligibility criteria was included until a sample of $n=200$ was reached in each district. The data collection tool/questionnaire is attached as a supplementary file.

\section{Measures}

All participants completed a paper/pencil questionnaire. The study collected demographic information about the participants, including age, gender (female, male, other), religious affiliation (Christian, Muslim, other), and educational attainment (primary, secondary, tertiary, other). The questionnaire contained the following constructs and items:

Sources of vaccination information and trust levels: We assessed the caregivers' sources of vaccination information (healthcare workers, family members, religious groups or places of worship, social media, and community members). Trust in each of the above sources was measured with a rating scale from 1 = "Not at all" to $5=$ "I totally trust."

Knowledge of HPV/cervical cancer. Knowledge of cervical cancer and HPV was tested with "Have you ever heard about cervical cancer and the HPV vaccine?", "Have you heard of the virus that causes cervical cancer?", and "Do you know that cervical cancer can be prevented?" These were answered with "yes" or "no."

5C psychological antecedents of vaccination: The 5C measured the confidence, complacency, constraints, calculation, and collective responsibility variables. Religion, rumor and masculinity as stand-alone additional variables were similarly measured, resulting in $5 \mathrm{C}+$. All items were measured on a scale ranging from $1=$ "strongly disagree" to $5=$ "strongly agree". The $5 \mathrm{C}+$ were measured as follows:

Confidence: "I am completely confident that vaccines are safe," "Vaccinations are effective," and "Regarding childhood vaccines, I am confident that public authorities decide in the best interest of the community."

Complacency: "Vaccination is unnecessary because vaccine-preventable diseases are not common anymore," "My child's immune system is so strong it also protects against diseases," and "Vaccine-preventable diseases are not so severe that I should vaccinate my child."

Constraints: "Everyday stress prevents me from getting my child vaccinated," "For me, it is inconvenient to have my child vaccinated," and "Visiting the doctor makes me feel uncomfortable; this keeps me from having my child vaccinated."

Calculation: "When I think about getting my child vaccinated, I weigh benefits and risks to make the best decision possible," "For each and every vaccination, I closely consider whether it is useful for my child," and "It is important for me to fully understand the topic of vaccination before I have my child vaccinated."

Collective Responsibility: "When everyone is vaccinated, I don't have to vaccinate my child, too," "I have my child vaccinated so my child can also protect people with a weaker immune system," and "Vaccination is a collective action to prevent the spread of diseases." 
For the above, a response scale ranging from "strongly disagree" to "strongly agree" was used. Religion: This was measured by "My religion does not support vaccination," and the response scale was from "strongly disagree" to "strongly agree."

Masculinity: The measurement item was "My husband's approval is important to vaccinate our child" using a scale from "strongly disagree" to "strongly agree."

Rumor/Misinformation: Using a scale from "strongly disagree" to "strongly agree", this variable assessed conspiratorial and belief elements such as "Vaccination against HPV promotes premarital sex," "Vaccination is a means to reduce our population," and "The HPV vaccine is meant to make our girls unable to have children in future, and prayers are an effective way to prevent vaccinepreventable diseases."

Vaccination intention: The intention to vaccinate for RI and HPV was measured by one item each: "I definitely intend to vaccinate my child when the next vaccination appointment is due" and "I will vaccinate my daughter against HPV in the future." Responses ranged from "strongly disagree" to "strongly agree."

\section{Data analysis}

The data were analyzed using R (version 3.6.3). Multiple regression analyses were performed to investigate if and how the $5 \mathrm{C}+$ influenced the caregivers' intention to vaccinate children for RI and HPV and their knowledge about HPV and cervical cancer.

\section{Ethical considerations}

Ethical clearance and approval from the National Health Sciences Research Committee (ref. no.: 20/04/2544) was obtained before commencing data collection. Authorization for the study was also received from the Malawian Ministry of Health (Ref. no. MED/1/3). Informed written consent was obtained from all subjects prior to their participation.

\section{Results}

\section{Demographic characteristics}

The participants were 18 years or older (18-24 years: $18 \%$; $25-34$ years: $40 \% ; 35-45$ years: $33 \%$; $45-60$ years: $8 \%$; 60 years and older: 1\%); 483 participants were female, and 114 were male. The majority indicated being Christian (75\%) or Muslim (22\%). Few indicated believing in traditional religion ( $1 \%$ ) or not being religious at all (2\%). In terms of education, $11 \%$ had no formal education, $38 \%$ had completed primary education, $29 \%$ had completed secondary education and $4 \%$ had completed tertiary education.

\section{Sources of vaccination information}

About $82 \%$ of the respondents relied on healthcare workers for vaccination information, while $7 \%$ indicated friends and $4 \%$ family members as the most important source for vaccination information. Other sources, such as social media (Facebook, Twitter) and places of religious worship (mosque, church), were indicated by $1 \%$ or less. When asked about the accuracy of or trust in vaccination information provided by the different sources, trust in the community $(M=4.59, S D=0.90)$ and healthcare workers $(M$ $=4.30, S D=1.32)$ was high. Trust in information received from friends and family $(M=1.64, S D=1.23)$, social media $(M=1.32, S D$ $=0.89)$, and religious organizations $(M=1.65, S D=1.25)$ was rather low.

\section{C psychological antecedents of vaccination}

Each dimension of the original $5 \mathrm{C}$ included three items to be averaged. However, internal consistency was unexpectedly low for the five scales, with Cronbach's alpha ranging between -0.16 for collective responsibility and 0.4 for constraints. The maximum intercorrelation between two single items was $r=.41$ (for visiting the doctor makes me feel uncomfortable and own religion does not support vaccination). Consequently, we refrained from calculating any scale means and instead included all items separately in the subsequent analysis. Table 1 provides means $(M)$ and standard deviations ( $S D$ s) for all items, including the religion, rumors, and masculinity extensions $(5 \mathrm{C}+)$. 
Mean agreement with $5 \mathrm{C}+$ antecedents of vaccination, per item

\begin{tabular}{|c|c|c|}
\hline Variable & $M$ & $S D$ \\
\hline \multicolumn{3}{|l|}{ Confidence } \\
\hline Vaccines are safe & 4.19 & 1.27 \\
\hline Vaccinations are effective & 4.30 & 1.25 \\
\hline Public authorities decide in the best interest of the community & 4.06 & 1.32 \\
\hline \multicolumn{3}{|l|}{ Complacency } \\
\hline Vaccination-preventable diseases are not common anymore & 1.94 & 1.26 \\
\hline Immune system protects against diseases & 2.66 & 2.64 \\
\hline Vaccine-preventable diseases are not severe & 1.91 & 1.20 \\
\hline \multicolumn{3}{|l|}{ Constraints } \\
\hline Everyday stress prevents vaccination & 2.47 & 1.49 \\
\hline Receiving vaccinations is inconvenient & 2.40 & 1.40 \\
\hline Visiting the doctor makes me feel uncomfortable & 2.05 & 1.20 \\
\hline \multicolumn{3}{|l|}{ Calculation } \\
\hline Weighing benefits and risks & 3.52 & 2.70 \\
\hline Considering usefulness of vaccination & 3.97 & 1.39 \\
\hline Topic of vaccination must be fully understood & 4.32 & 1.20 \\
\hline \multicolumn{3}{|l|}{ Collective responsibility } \\
\hline No need for vaccination when everyone is vaccinated & 1.96 & 1.21 \\
\hline Vaccinated to protect people with weaker immune system & 2.45 & 1.50 \\
\hline Vaccination as collective action to prevent the spread of diseases & 4.33 & 1.57 \\
\hline \multicolumn{3}{|l|}{ Religion } \\
\hline Own religion does not support vaccination & 1.88 & 1.02 \\
\hline \multicolumn{3}{|l|}{ Masculinity } \\
\hline Husband's approval important for vaccination & 3.19 & 1.56 \\
\hline \multicolumn{3}{|l|}{ Rumors } \\
\hline Vaccination causes infertility & 2.40 & 1.48 \\
\hline Prayers prevent measles & 2.76 & 1.54 \\
\hline Vaccination is means to reduce population & 2.22 & 1.50 \\
\hline
\end{tabular}

\section{Determinants of caregiver's intention to vaccinate child}

Explorative backward elimination regression analyses were performed to determine the relevant demographic and psychological variables related to vaccination intention. First, the $5 \mathrm{C}+$ were considered predictors of the intention to vaccinate one's child when 
the next appointment is due. The backward elimination regression algorithm removed those items that did not explain variance in intentions, resulting in a model with nine predictors (Table 2). Confidence in vaccine safety was the strongest predictor of vaccination intention, followed by the constraint of everyday stress. Stronger agreement that the topic of vaccination must be fully understood was associated with higher vaccination intentions, while items from the collective responsibility subscale did not play a role. For the complacency items, inconsistent results emerged. Vaccination intentions were lower for those thinking that the child's immune system protects against diseases but higher for those believing that vaccine-preventable diseases are not severe. The importance of a husband's approval increased vaccination intention.

Table 2

Backward elimination regression results for caregiver's intentions to vaccinate children

\begin{tabular}{|llllll|}
\hline Predictor & $\mathbf{b}$ & $\mathbf{B}$ & $\mathbf{S E}$ & $\mathbf{C l}-$ & $\mathbf{C l +}$ \\
\hline (Constant) & & -0.43 & 0.38 & -1.180 & 0.322 \\
\hline Confidence: Vaccines are safe & $\mathbf{0 . 5 2}$ & $\mathbf{0 . 6 4}$ & 0.05 & 0.544 & 0.737 \\
\hline Confidence: Vaccinations are effective & $\mathbf{0 . 1 0}$ & $\mathbf{0 . 1 4}$ & 0.05 & 0.035 & 0.236 \\
\hline Complacency: Child's immune system protects against diseases & $\mathbf{- 0 . 1 2}$ & $\mathbf{- 0 . 0 6}$ & 0.02 & -0.105 & -0.022 \\
\hline Complacency: Vaccine-preventable diseases are not severe & $\mathbf{0 . 1 0}$ & $\mathbf{0 . 1 3}$ & 0.06 & 0.029 & 0.249 \\
\hline Constraints: Everyday stress prevents vaccination & $\mathbf{0 . 1 6}$ & $\mathbf{0 . 1 7}$ & 0.04 & 0.087 & 0.260 \\
\hline Constraints: Visiting the doctor makes me feel uncomfortable & $\mathbf{- 0 . 0 8}$ & $\mathbf{- 0 . 1 1}$ & 0.05 & -0.210 & -0.002 \\
\hline Calculation: Weighing benefits and risks & -0.05 & -0.03 & 0.02 & -0.071 & 0.010 \\
\hline Calculation: Topic of vaccination must be fully understood & $\mathbf{0 . 0 9}$ & $\mathbf{0 . 1 4}$ & 0.06 & 0.025 & 0.247 \\
\hline Masculinity: Husband's approval important for vaccination & $\mathbf{0 . 0 8}$ & $\mathbf{0 . 0 9}$ & 0.04 & 0.009 & 0.167 \\
\hline
\end{tabular}

Note. $\mathrm{R}^{2}=.41$; adjusted $\mathrm{R}^{2}=.40$. Bold values are significant at $p<.05 . \mathrm{Cl}$ and $\mathrm{Cl}+$ are the lower and upper bounds of the $95 \%$ confidence intervals.

Second, for all significant variables, backward elimination regression analyses were performed to explore factors predicting higher or lower levels of confidence, constraints, etc. Demographic variables such as age, gender, religion, education, and employment, as well as belief in rumors (about prayers preventing measles, vaccinations being a means to reduce the population, and HPV vaccine ruining fertility) and trust in healthcare workers were considered predictors (Table 3). Participants had lower confidence in vaccine safety when they believed in rumors and misinformation, such as prayers preventing measles and the HPV vaccine ruining fertility. Confidence in vaccine effectiveness was also affected by misinformation and was lower for unemployed participants but higher for those whose youngest child was male and who had more trust in healthcare workers. For belief about the child's immune system protecting against diseases, none of the given predictors was significant. On the contrary, thinking that vaccine-preventable diseases are not severe was affected by multiple variables: older adults (35-60 years), males, those believing in traditional religions, and those agreeing with the rumor that vaccination is a means to reduce the population. Participants whose youngest child was male, who had at least secondary education, and who thought that prayers prevent measles indicated more strongly that everyday stress prevented them from vaccinating their child. Visiting the doctor made the participants feel more uncomfortable if they believed in traditional religion and misinformation (i.e., that vaccination is a means to reduce the population and that the HPV vaccine ruins fertility) and if they had lower trust in healthcare workers. Those who trusted healthcare workers also agreed with the statement that the topic of vaccination must be fully understood. A husband's approval for vaccination was more important for participants who had primary or secondary education, more trust in healthcare workers, and stronger belief that vaccination is a means to reduce the population and that the HPV vaccine ruins fertility.

Table 3 Backward elimination regression results predicting relevant psychological antecedents of vaccine hesitancy 


\begin{tabular}{|c|c|c|c|c|c|c|c|c|}
\hline \multirow[t]{2}{*}{ Predictor } & \multicolumn{2}{|c|}{ Confidence } & \multicolumn{2}{|c|}{ Complacency } & \multicolumn{2}{|c|}{ Constraints } & \multirow{2}{*}{$\begin{array}{c}\text { Calculation } \\
\text { Topic of } \\
\text { vaccination must } \\
\text { be fully } \\
\text { understood }\end{array}$} & \multirow{2}{*}{$\begin{array}{c}\text { Masculinity } \\
\text { Husband's } \\
\text { approval } \\
\text { important for } \\
\text { vaccination }\end{array}$} \\
\hline & $\begin{array}{l}\text { Vaccines are } \\
\text { safe }\end{array}$ & $\begin{array}{l}\text { Vaccines are } \\
\text { effective }\end{array}$ & $\begin{array}{l}\text { Child's immune } \\
\text { system protects } \\
\text { against diseases }\end{array}$ & $\begin{array}{c}\text { Vaccine- } \\
\text { preventable } \\
\text { diseases are not } \\
\text { severe }\end{array}$ & $\begin{array}{l}\text { Everyday stress } \\
\text { prevents } \\
\text { vaccination }\end{array}$ & $\begin{array}{c}\text { Visiting the } \\
\text { doctor makes me } \\
\text { feel } \\
\text { uncomfortable }\end{array}$ & & \\
\hline (Constant) & $4.97(0.14)^{* * *}$ & $4.01(0.27)^{* * *}$ & $2.68(0.14)^{* * *}$ & $1.15(0.42)^{* *}$ & $2.62(0.61)^{* * *}$ & $1.86(0.47)^{* * *}$ & $3.78(0.18)^{* * *}$ & $1.33(0.65)^{*}$ \\
\hline \multicolumn{9}{|l|}{ Age (Baseline: 18-24) } \\
\hline $25-34$ & & & & $-0.26(0.16)$ & & & & \\
\hline $35-44$ & & & & $-0.51(0.17)^{* *}$ & & & & \\
\hline $45-60$ & & & & $-0.75(0.25)^{* *}$ & & & & \\
\hline $60+$ & & & & $-0.77(0.66)$ & & & & \\
\hline $\begin{array}{l}\text { Gender: male (Baseline: } \\
\text { female) }\end{array}$ & & & & $0.40(0.15)^{* *}$ & & & & \\
\hline $\begin{array}{l}\text { Gender of youngest child: } \\
\text { male (Baseline: female) }\end{array}$ & & $0.36(0.12) * *$ & & & $0.28(0.14)^{*}$ & & & \\
\hline \multicolumn{9}{|l|}{$\begin{array}{l}\text { Religion: (Baseline: not } \\
\text { religious) }\end{array}$} \\
\hline Christian & & & & $0.66(0.40)$ & $-0.84(0.51)$ & $0.13(0.41)$ & & $-0.09(0.54)$ \\
\hline Muslim & & & & $0.75(0.41)$ & $-0.31(0.53)$ & $0.26(0.43)$ & & $0.30(0.56)$ \\
\hline Traditional & & & & $1.79(0.63)^{* *}$ & $-0.09(0.82)$ & $1.71(0.67)^{*}$ & & $1.34(0.88)$ \\
\hline Other & & & & $0.19(1.17)$ & $-2.41(1.52)$ & $-1.09(1.24)$ & & $-2.51(1.62)$ \\
\hline \multicolumn{9}{|l|}{$\begin{array}{l}\text { Education (Baseline: no } \\
\text { formal education) }\end{array}$} \\
\hline Primary education & & & & & $0.42(0.22)$ & & & $0.48(0.23) *$ \\
\hline Secondary education & & & & & $0.62(0.23)^{* *}$ & & & $0.63(0.25)^{*}$ \\
\hline Tertiary education & & & & & $1.14(0.46) *$ & & & $0.90(0.49)$ \\
\hline Being unemployed & & $-0.27(0.12) *$ & & $-0.22(0.11)$ & & $-0.22(0.12)$ & & $0.25(0.16)$ \\
\hline Trust in healthcare workers & & $0.09(0.05)^{*}$ & & & $-0.10(0.05)$ & $-0.10(0.05)^{*}$ & $0.14(0.04)^{* * *}$ & $0.14(0.06)^{*}$ \\
\hline \multicolumn{9}{|l|}{ Believing in rumors: } \\
\hline $\begin{array}{l}\text { Vaccination is means to } \\
\text { reduce population }\end{array}$ & & & & $0.18(0.04)^{* * *}$ & & $0.12(0.04)^{* *}$ & & $0.12(0.05)^{*}$ \\
\hline Prayers prevent measles & $-0.11(0.04) *$ & $0.07(0.04)$ & & & $0.13(0.05)^{* *}$ & & & \\
\hline $\begin{array}{l}\text { HPV vaccine ruins } \\
\text { fertility }\end{array}$ & $-0.22(0.05) * * *$ & $-0.16(0.05)^{* * *}$ & & & & $0.12(0.04) * *$ & & $0.14(0.06)^{*}$ \\
\hline
\end{tabular}

Note. Estimates (b, SE in parentheses). Significance indicators: * $.05>\star \star \geq .01>\star \star \star \geq .001>$

\section{HPV knowledge}

Most participants ( $n=521$ vs. $n=67: 87 \%$ vs. $11 \%$ ) indicated having heard about cervical cancer. About two-thirds had heard about human papillomavirus ( $n=406$ vs. $n=174: 68 \%$ vs. $29 \%$ ) and knew that the cancer could be prevented ( $n=420$ vs. $n=159: 70 \%$ vs. $27 \%$ ). However, only about one-fifth knew about the HPV virus ( $n=130$ vs. $n=453: 22 \%$ vs. $76 \%$ ). By aggregating the four knowledge indicators, the mean HPV knowledge was $0.64(S D=0.30)$.

Again, a backward elimination regression analysis was performed to identify the variables related to HPV knowledge. Age, gender, religion, education, employment, belief in rumors/misinformation (i.e., believing that vaccination is a means to reduce the population, HPV vaccines reduce girls' fertility, and vaccination against HPV promotes premarital sex), and trust in healthcare workers were considered predictors of mean HPV knowledge (Table 4). Religion and education were major drivers of HPV knowledge. Those believing in traditional African religion had lower HPV knowledge compared to those who were not religious, and respondents who had completed secondary or tertiary education showed higher knowledge than those without formal education. With regards to rumors/misinformation, believing that HPV vaccines impact fertility increased HPV knowledge. 
Standardized regression results predicting mean HPV knowledge

\begin{tabular}{|c|c|c|c|c|c|}
\hline Predictor & B & b & SE & $\mathrm{Cl}-$ & $\mathrm{Cl}+$ \\
\hline (Constant) & & 0.64 & 0.10 & 0.44 & 0.84 \\
\hline Gender: male (Baseline: female) & -0.07 & -0.05 & 0.03 & -0.11 & 0.02 \\
\hline \multicolumn{6}{|l|}{ Religion (Baseline: not religious) } \\
\hline Christian & -0.15 & -0.10 & 0.10 & -0.29 & 0.09 \\
\hline Muslim & -0.25 & -0.18 & 0.10 & -0.38 & 0.01 \\
\hline Traditional & -0.18 & -0.43 & 0.14 & -0.71 & -0.16 \\
\hline Other & 0.00 & 0.00 & 0.22 & -0.44 & 0.44 \\
\hline \multicolumn{6}{|l|}{ Education (Baseline: no formal education) } \\
\hline Primary education & 0.08 & 0.05 & 0.04 & -0.03 & 0.13 \\
\hline Secondary education & 0.28 & 0.18 & 0.04 & 0.10 & 0.27 \\
\hline Tertiary education & 0.11 & 0.17 & 0.08 & 0.02 & 0.32 \\
\hline Believing in rumor: HPV vaccine ruins fertility & 0.10 & 0.02 & 0.01 & 0.00 & 0.04 \\
\hline
\end{tabular}

\section{Determinants of intention to vaccinate daughters against HPV}

A backward elimination regression analysis was performed to determine the demographic and psychological variables related to HPV vaccination intention. Age, religion, education, employment, gender, HPV knowledge, the 5C+, rumors/misinformation, and trust in healthcare workers were considered predictors of the intention to vaccinate daughters against HPV. Compared to the previous regression predicting intention to vaccinate the youngest child, the gender of the youngest child and the belief that prayers prevent measles were not included here, as the analyses focused on daughters above 9 years of age and HPV. For the analysis, respondents were excluded if they had no daughter above 9 years of age or if she had already received the HPV vaccine, resulting in a reduced sample of $n=133$.

Table 5 shows the standardized regression results. Of all the demographic variables, only education influenced vaccination intention. Respondents with a secondary or tertiary education showed lower intentions than those with no formal or primary education. HPV vaccination intentions increased with trust in healthcare workers but decreased with higher confidence in the safety of the vaccine and the system delivering it. When respondents thought that their children's immune systems protected against diseases, vaccination intentions increased. Similar effects could be observed for indicators of calculation and collective responsibility: weighing risks and benefits and thinking that vaccination protects people with weaker immune systems increased intentions to get adolescent daughters vaccinated against HPV. Husbands' approval also had a positive effect on vaccination intentions. Interestingly, for knowledge about HPV and its prevention, no direct effect on vaccination intentions could be found. 
Table 5

Standardized backward elimination regression results predicting intentions to get adolescent daughters vaccinated against HPV

\begin{tabular}{|c|c|c|c|c|c|}
\hline Predictor & B & b & SE & $\mathrm{Cl}-$ & $\mathrm{Cl+}$ \\
\hline (Constant) & & 2.93 & 1.07 & 0.83 & 5.03 \\
\hline \multicolumn{6}{|l|}{ Age (versus 18-24) } \\
\hline $25-34$ & -0.30 & -1.08 & 0.73 & -2.50 & 0.35 \\
\hline $35-44$ & -0.09 & -0.32 & 0.73 & -1.76 & 1.12 \\
\hline $45-60$ & -0.07 & -0.38 & 0.80 & -1.96 & 1.20 \\
\hline \multicolumn{6}{|l|}{ Education (versus no formal education) } \\
\hline Primary education & -0.21 & -0.74 & 0.44 & -1.61 & 0.12 \\
\hline Secondary education & -0.33 & -1.25 & 0.48 & -2.19 & -0.31 \\
\hline Tertiary education & -0.22 & -4.56 & 1.61 & -7.72 & -1.40 \\
\hline Trust in healthcare workers for accurate vaccination information & 0.19 & 0.24 & 0.10 & 0.05 & 0.43 \\
\hline Confidence: Vaccines are safe & -0.17 & -0.21 & 0.10 & -0.41 & 0.00 \\
\hline Confidence: Public authorities decide in the best interest of the community & -0.24 & -0.32 & 0.11 & -0.53 & -0.11 \\
\hline Complacency: Immune system protects against diseases & 0.24 & 0.30 & 0.10 & 0.11 & 0.50 \\
\hline Calculation: Weighing benefits and risks & 0.24 & 0.28 & 0.09 & 0.11 & 0.45 \\
\hline Collective responsibility: Vaccinate to protect people with weaker immune system & 0.27 & 0.31 & 0.09 & 0.13 & 0.49 \\
\hline Masculinity: Husband's approval important for vaccination & 0.27 & 0.23 & 0.09 & 0.06 & 0.40 \\
\hline
\end{tabular}

\section{Belief in rumors}

Since belief in rumors and misinformation (i.e., that prayers prevent measles, vaccines are a means to reduce the population, and the HPV vaccine ruins girls' fertility) was associated with both general intentions to vaccinate children and HPV knowledge, an additional backward elimination regression was performed to identify the characteristics associated with those beliefs. A mean belief in rumors was first calculated from the three items $(M=2.46, S D=1.17$, Cronbach's alpha $=0.67)$. Age, gender, religion, education, employment, and trust in healthcare workers, social media, religion, friends, family, and the local community were considered predictors of mean belief. As indicated by the standardized regression results (Table 6), age was a significant predictor: compared to the youngest group of participants (18-24 years), respondents aged 25-34 years old indicated a higher belief in rumors. For older participants, beliefs decreased, with the oldest participants $(60+$ years $)$ showing the least belief. Being unemployed increased the average belief in rumors, while trust in healthcare workers reduced it. No effects could be found for religion, education, gender, and trust in other sources of information, such as social media or friends and family. 
Standardized backwards regression predicting average belief in rumors

\begin{tabular}{|c|c|c|c|c|c|}
\hline Predictor & $\beta$ & b & SE & $\mathrm{Cl}-$ & $\mathrm{Cl}+$ \\
\hline Age (versus 18-24) & & 3.18 & 0.20 & 2.79 & 3.57 \\
\hline $25-34$ & 0.15 & 0.36 & 0.13 & 0.09 & 0.62 \\
\hline $35-44$ & 0.10 & 0.24 & 0.14 & -0.04 & 0.51 \\
\hline $45-60$ & -0.05 & -0.22 & 0.20 & -0.61 & 0.18 \\
\hline $60+$ & -0.09 & -1.36 & 0.64 & -2.62 & -0.09 \\
\hline Being unemployed & 0.10 & 0.25 & 0.10 & 0.06 & 0.44 \\
\hline Trust in healthcare workers for accurate vaccination information & -0.28 & -0.24 & 0.03 & -0.31 & -0.17 \\
\hline
\end{tabular}

\section{Discussion}

Based on the results, caregivers' vaccination acceptance for RI is motivated predominantly by aspects related to confidence in vaccine safety, followed by everyday stress (constraints; an unexpected relation) and some minor influences by variables such as vaccine effectiveness (confidence), beliefs in the child's immune system (complacency), or husband's approval (masculinity). Other variables that correlated with the intention to vaccinate one's child were the feeling that vaccine-preventable diseases are not severe (complacency), fear of doctors (constraints), and risk-benefit analysis and understanding vaccine topics (calculation).

In sum, confidence in vaccine safety was the strongest predictor for vaccination intention. Confidence in vaccines did not differ according to demographic characteristics but was strongly related to rumors (i.e., that prayers prevent measles; and HPV vaccine ruins fertility). A further analysis demonstrated that confidence in vaccine safety decreased when people believed that prayers or religious rituals (e.g., taking "holy communion") could prevent or serve as prophylaxis against vaccine-preventable diseases such as measles.

It is not just the safety of vaccines that worries caregivers about RI but also whether vaccines are effective. Assessing this alongside other variables showed that confidence in vaccine effectiveness increased with trust in vaccination information provided by healthcare workers but decreased if the participants were unemployed or thought that the HPV vaccine reduces fertility. Therefore, the vaccination intention for RI decreased among Malawian caregivers due to concerns about vaccine safety and effectiveness. For the HPV vaccine, decreased intention to vaccinate a daughter against cervical cancer was traced to belief in rumors and the caregiver's lack of confidence in the ability of the public authorities to decide in the best interest of the populace.

Belief in rumors and being unemployed had a negative effect on safety perception; therefore, believing in these rumors and not having a job both decreased vaccination intention, hence increasing vaccine hesitancy.

Addressing vaccine hesitancy based on rumors by using health promotion campaigns to increase vaccination demand requires a well-tailored and specific communication strategy. The strategy should not only debunk rumors surrounding vaccines such as HPV but also resolve vaccine safety and effectiveness concerns raised by caregivers about vaccination in general. Also, since unemployment plays a significant role in vaccine hesitancy, incentives could be built around vaccination attendance in health facilities, in the form of provision of lunch and transportation reimbursement for caregivers, besides maintaining a free vaccination programme. Incentivizing unemployed caregivers will mitigate constraints that accompany out-of-pocket costs during vaccination. This could help to avoid complacency and dropout.

Confidence in vaccine safety and effectiveness are global vaccine hesitancy phenomena, especially in high-income countries where studies and countermeasures are exhaustive $[44,70]$. However, low-income settings such as Malawi will presumably require a more bottom-top approach, since the majority of its population lives in rural areas. Also, since the caregivers in this study trusted 
healthcare workers most for their vaccination information, the Expanded Program on Immunization (EPI) in Malawi should integrate local healthcare workers into the heart of vaccination education.

Complacent behavior among caregivers was a unique finding. The perception that vaccine-preventable diseases are not so severe or not perceiving diseases as high risk and vaccination as necessary was predominantly found among the older population (35$60)$ compared to the young adults (18-34). One would expect that the older people become, the higher their health-seeking behavior or self-efficacy attached to preventive behavior and vulnerability to diseases $[71,72]$. This is also contrary to expectations that younger individuals have higher risk-seeking and invulnerability behavior due to feelings of positive subjective personal health status [71, 73, 74]. As complacency correlates with a positive general risk attitude, risk-seeking behaviors are usually expected [49, 75]. However, it is remarkable to find this among the older population instead of the younger. Perhaps they thought that these childhood diseases are not relevant for them. Further studies may be needed to understand the reasons for this trend, and a targeted intervention at the older population on the severity of vaccine-preventable diseases, preferably using negative framing, is recommended.

The role of education was notable because education mostly correlates with positive attitudes and behavior toward vaccination [76-78]. The opposite, as in the case of this study, is not frequently found. However, Malawi may not necessarily be the only country with this finding. The influence of education on childhood immunization uptake in Spain was found to be unrelated: the less educated parents had higher childhood immunization rates [79]. Similarly, when a state of emergency was declared in Washington state in 2019 due to a measles outbreak, affluent and well-educated parents were most hesitant to vaccinate children [80]. Therefore, communication messages that target risk perception among urban population is an appropriate intervention.

Rumor/misinformation has hitherto been under-estimated in various vaccine demand creation interventions across the SSA region $[68,81]$, and it is an important revelation of this study. There was a strong relationship between rumors and vaccination intention among caregivers for RI and HPV knowledge, and belief in rumors was a major barrier against HPV vaccination among caregivers of adolescent girls in Malawi.

Beliefs in rumors and misinformation assessed in the study were associated with general knowledge of HPV and vaccination intention. A closer analysis showed that being young, unemployed, or having low trust in healthcare workers increased those beliefs. The devastating impacts of rumors on vaccination demand cannot be overstated. While being unemployed and having low trust in healthcare workers as drivers for low vaccination intention is not unexpected, in view of the social baggage that comes with it when analysed. But being young contributing to higher belief in rumors is a surprise and needs to be further investigated.

Although several factors could influence this, including young people's exposure to social media misinformation campaigns, where the bulk of vaccination misinformation is currently peddled [82-85].

A tailored strategy that employs scientific research and communicates in an evidence-informed manner to inspire recurrent stakeholder dialogue by raising different voices to allow for discussions is needed. This process builds partnerships using facts and, at the same time, counters misinformation with a non-aggressive posture. Therefore, while social media use has been associated with a negative impact on public perception of vaccines and vaccinations, it also presents a unique opportunity to aggressively disseminate scientific evidence through the same platform. This could be how groups of young vaccine misinformation spreaders can be reached and convinced that rumors are wrong, using evidence. Also, although knowledge deficits arising from lack of cognitive information or affective or psychomotor abilities needed for positive health-seeking behavior seem apparent in Malawi, based on the results, when knowledge is confounded by education (especially little or no formal education), improving knowledge deficits among this group is not enough. Intervention geared toward addressing this must feel appropriate and fit people's value systems and cultural norms.

Trust is the bedrock of vaccination acceptance. Therefore, trust must be built in and around all facets of national EPI programs, including vaccine development, distribution, policies, healthcare systems (doctors, nurses, and immunizers), and mass vaccination campaigns. For vaccination acceptance strategies to succeed, efforts should focus first on building trust, improving vaccine confidence, and dispelling rumors associated with vaccines or vaccinations.

Husbands or fathers were identified in this study as crucial to childhood vaccination uptake. A father's consent for childhood vaccination had a significant positive effect on vaccination intentions. Even more so, a husband's or father's approval is important 
for women with little education (primary or secondary education), little trust in healthcare workers, and those who believe in rumors. Thus, interventions should focus on improving men's positive perceptions of vaccines and the need to improve vaccination uptake in Malawi. Encouraging men to attend immunization activities, like ante-natal initiatives, would be a positive step toward improving perception.

\section{Conclusions}

Overall, the $5 \mathrm{C}$ psychological antecedents model for measurement of vaccine hesitancy in Malawi was very effective, although not completely adaptable, as it was initially developed and used in high-income-countries. The results, plus its extension show that items measuring aspects of confidence, complacency, constraints, calculation, and masculinity were the significant factors that drive low vaccination uptake for RI. For the HPV vaccination, rumors, lack of trust in government, education level, and husband's approval to vaccinate daughters played predominant roles. Thus, addressing vaccine hesitancy in Malawi requires a multidimensional approach that involves both communication tools and devolution of management of vaccination programs to the local level, led by local healthcare workers, since trust in them is high among the population. However, further studies may be needed to understand low-risk perceptions among the older population and the potential role of local non-governmental organizations in building trust in vaccines.

\section{Abbreviations}

EPI: Expanded Program on Immunization

HPV: Human papillomavirus

RI: Routine Immunization

SAGE: The Strategic Advisory Group of Experts (SAGE) on Immunization

SSA: Sub-Saharan Africa

UNICEF: United Nations International Children's Emergency Fund

WHO: World Health Organization

\section{Declarations}

\section{Ethics approval and consent to participate}

Ethical clearance from relevant authorities was obtained, including ethics approval from the National Health Sciences Research Committee in Malawi (reference number 20/04/2544) and authorization from the Malawi Ministry of Health (reference number MED/1/3). Written informed consent was obtained from all subjects. The study and methods therein were performed in accordance with the relevant guidelines and regulations.

\section{Consent for publication}

Not applicable.

\section{Availability of data and materials}

The materials/dataset used and/or analysed are available at the Open Science Framework https://osf.io/pzaer/

\section{Competing interests}

The authors declare that they have no competing interests.

Funding

Page 14/19 
This work was supported by an unconditional educational grant from Merck Sharp \& Dohme (MSD) through the Agence de Médecine Préventive Afrique. However, they had no influence on the study design, data collection and analysis, decision to publish, or preparation of the manuscript.

\section{Authors' contributions}

Conceptualization: GCA, CB, and TAE.

Methodology: GCA, CB, and TAE.

Investigation: GCA and TAE.

Results analysis: GCA, CB, and PS.

Writing-original draft: GCA, CB, and TAE.

Writing-review \& editing: GCA, PS, CB, and TAE.

All authors have read and approved the final manuscript.

\section{Acknowledgements}

This study acknowledges the support of the Malawi Ministry of Health and the management and staff of the Expanded Program on Immunization (EPI): Dr. Mike Nenani Chisema (Malawi EPI Country Manager), Temwa Mzengeza (Malawi EPI National Routine Immunization Officer), Mr. Luciano Njoka Abraham (Salima District EPI Coordinator), and the EPI district stakeholders in the Salima, Dowa, and Zomba districts. Finally, many thanks go to Dr. Abdu A. Adamu (Nigeria) and the health facilities in Malawi used for data collection, including Salima District Hospital, Khombedza Health Center, Dowa District Hospital, Dzaleka Health Center, Zomba District Hospital, Matawale Health Center, Bwaila Hospital, and Area 15 Health Center.

\section{References}

1. Oyo-Ita A, Wiysonge CS, Oringanje C, Nwachukwu CE, Oduwole O, Meremikwu MM. "Interventions for improving coverage of childhood immunisation in low- and middle-income countries". Cochrane database Syst Rev. Wiley-Blackwell; 2016;7(7):CD008145.

2. European Centre for Disease Prevention and Control (ECDC). Catalogue of interventions addressing vaccine hesitancy. Stockholm: ECDC; 2017.

3. UNICEF Report: Immunization, July 2019. Accessed October 19, 2020. https://data.unicef.org/topic/childhealth/immunization/

4. Gulland, Anne. Africa set to be declared polio free as Nigeria marks three years without the disease. The Telegraph; May 20, 2019. (Assessed on Jan. 15, 2021). https://www.telegraph.co.uk/global-health/science-and-disease/africa-set-declared-poliofree-nigeria-marks-three-years-without/

5. World Health Organization (WHO).Global Immunization Vision and Strategy (GIVS): 2006-2015. World Health Organization. 2005. https://apps.who.int/iris/handle/10665/69146

6. Kamara, Lidija., Lydon, Patrick., Bilous, Julian., Vandelaer, Jos., Eggers, Rudi., Gacic-Dobo, Marta., Meaney, William., Okwo-Bele, Jean-Marie. Global Immunization Vision and Strategy (GIVS): a mid-term analysis of progress in 50 countries, Health Policy and Planning. 2013; 28 (1): 11-19, https://doi.org/10.1093/heapol/czs020

7. World Health Organization. Immunization, vaccines and biologicals-data, statistics and graphs. Geneva, Switzerland: World Health Organization; 2018. https://www.who.int/immunization/monitoring_surveillance/data/en/

8. Global Alliance for Vaccine and Immunization (GAVI). Facts \& Figures: Statistics measuring our impact on global immunization. Feb. 2021. https://www.gavi.org/sites/default/files/document/2021/Gavi-Facts-and-figures-February-21.pdf (Accessed May 17, 2021).

9. Global Vaccine Action Plan 2011 - 2020 (GVAP). World Health Organization. 2013. 
10. Martin, Rebecca. On Behalf of the SAGE DoV GVAP Working Group. Decade of Vaccine Action Plan: Summary of the GVAP Implementation Progress. SAGE, Geneva, 2013.

11. Vanderslott, Samantha., Dadonaite, Bernadeta and Roser, Max. "Vaccination". 2019. Published online at OurWorldlnData.org. (Assessed January 2, 2020) https://ourworldindata.org/vaccination

12. WHO SAGE. National Immunization Technical Advisory Groups: Background Paper. World Health Organisation (WHO). April 2017. (Assessed December 28, 2020).

https://www.who.int/immunization/sage/meetings/2017/april/1_NITAGs_background_document_SAGE_April_2017.pdf

13. Okwo-Bele, Jean-Marie (2015, April 22). Together we can close the immunization gap. World Health Organisation (WHO).

14. WHO/UNICEF Estimates of National Immunization Coverage (WUENIC), July 2019 updated. https://apps.who.int/immunization_monitoring/globalsummary/timeseries/tswucoveragedtp3.html

15. LaFond A, Kanagat N, Steinglass R, Fields R, Sequeira J, Mookherji S. Drivers of routine immunization coverage improvement in Africa: findings from district-level case studies. Health Policy Plan. 2015;30(3):298-308.

16. Mihigo, Richard. Okeibunor, Joseph. Anya, Blanche. Mkanda, Pascal. Zawaira, Felicitas. "Challenges of immunization in the African Region." The Pan African medical journal.2017; 27, Suppl 3. doi:10.11604/pamj.supp.2017.27.3.12127

17. Tarantola D, Hacen M, Lwanga S, Clements CJ. Is Immunization Coverage in Africa Slipping? An Evaluation of Regional Progress to 2013. Ann Vaccines Immunization. 2014; 1(2): 1007.

18. World Health Organization. Immunization coverage: Key Challenges. July 15, 2020. https://www.who.int/news-room/factsheets/detail/immunization-coverage. (Accessed May 16, 2021).

19. Regional Office for Africa WHO. Experts caution against stagnation of immunization coverage in Africa. Brazzaville, Congo. 2019. https://www.afro.who.int/news/experts-caution-against-stagnation-immunization-coverage-africa (Accessed June 1 , 2021).

20. World Health Organization, UNICEF. Progress and Challenges with achieving Universal Immunization Coverage - 2018 WHO/UNICEF Estimates of National immunization Coverage. 2019. https://www.who.int/immunization/monitoring_surveillance/who-immuniz.pdf (Accessed June 1, 2021).

21. World Health Organization. Immunization. Vaccine hesitancy: A growing challenge for immunization programmes. Geneva. Aug. 18, 2015. https://www.who.int/news/item/18-08-2015-vaccine-hesitancy-a-growing-challenge-for-immunizationprogrammes (Accessed Nov. 29, 2020).

22. Larson, HJ., Jarrett, C., Eckersberger, E., Smith, DMD., Paterson, P. Understanding vaccine hesitancy around vaccines and vaccination from a global perspective: a systematic review of published literature, 2007-2012. Vaccine 2014; 32: $2150-59$.

23. National Statistical Office (NSO) [Malawi] and ICF. Malawi Demographic and Health Survey 2015-16. Zomba, Malawi, and Rockville, Maryland, USA. 2017.

24. Global Alliance for Vaccine and Immunization (GAVI) Annual Progress Report 2019.

25. Tsega, A., Hausi, H., Chriwa, G., Steinglass, R., Smith, D., \& Valle, M. Vaccination coverage and timely vaccination with valid doses in Malawi. Vaccine Reports, 2016; 6, 8-12. https://doi.org/10.1016/j.vacrep.2016.06.001

26. Bouassa, RS., Prazuck, T., Lethu, T., Meye, J.F., \& Bélec, L. Cervical cancer in sub-Saharan Africa: an emerging and preventable disease associated with oncogenic human papillomavirus. Medecine et Sante Tropicales, 2017; 27 (1), 16-22.

27. Bruni L, Albero G, Serrano B, Mena M, Gómez D, Muñoz J, Bosch FX, de Sanjosé S. Human Papillomavirus and Related Diseases in Africa. Summary Report, June 17, 2019. Pp. iv.

28. Ferlay J, Ervik M, Lam F, Colombet M, Mery L, Piñeros M, Znaor A, Soerjomataram I, Bray F. Global Cancer Observatory: Cancer Today. Lyon. 2018.

29. Bray F, Ferlay J, Soerjomataram I, Siegel RL, Torre LA, Jemal A. Global Cancer Statistics 2018: GLOBOCAN estimates of incidence and mortality worldwide for 36 cancers in 185 countries. Cancer Journal for Clinicians. 2018; 68(6): 394-424. DOI: 3322/caac. 21492 .

30. Msyamboza, KP., Dzamalala, C., Mdokwe, C., Kamiza, S., Lemerani, M., Dzowela, T., Kathyola, D. Burden of cancer in Malawi; common types, incidence and trends: national population-based cancer registry. BMC Res Notes. 2012; 16 (5):149. 
31. Maseko, FC., Chirwa, ML., Muula, AS. Cervical cancer control and prevention in Malawi: need for policy improvement. Pan African Medical Journal. 2015;22:247. [doi: 10.11604/pamj.2015.22.247.6439]

32. Fort, V.K., Makin, M.S., Siegler, A.J., Ault, K., Rochat, R. Barriers to cervical cancer screening in Mulanje, Malawi: A qualitative study. Patient Prefer Adher, 2011; 5, 125-131.

33. Maseko, FC., Chirwa, ML., Muula, AS. Client satisfaction with cervical cancer screening. BMC health services research. 2014: 14 (420). doi:10.1186/1472-6963-14-420

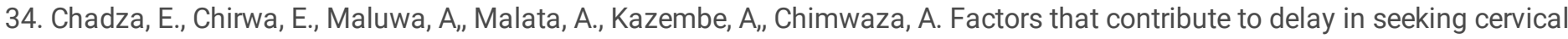
cancer diagnosis and treatment among women in Malawi. Health Aff. 2012;14(11):1015- 1022. doi:

10.4236/health.2012.411155.

35. Ashrafi, GH and Salman, NA. Pathogenesis of Human Papillomavirus - Immunological Responses to HPV Infection, Human Papillomavirus - Research in a Global Perspective, Rajamanickam Rajkumar, IntechOpen, 2016. DOI: 10.5772/63965. Available from: https://www.intechopen.com/books/human-papillomavirus-research-in-a-global-perspective/pathogenesis-of-humanpapillomavirus-immunological-responses-to-hpv-infection

36. Ault, Kevin A. Epidemiology and Natural History of Human Papillomavirus Infections in the Female Genital Tract. Infect Dis Obstet Gynecol. 2006: 40470. DOI: 10.1155/IDOG/2006/40470

37. Liu X-X, Fan X-L, Yu Y-P, Ji L, Yan J, Sun A-H. Human papillomavirus prevalence and type-distribution among women in Zhejiang Province, Southeast China: A cross-sectional study. BioMed Central; 2014;14(1):708.

38. Baseman, JG \& Koutsky, LA. The epidemiology of human papillomavirus infections. Journal of Clinical Virology. 2005; 32:1624.

39. Clair, K. Does Empowerment Predict Vaccine Uptake? Identifying Early Adopters of Recently Introduced Childhood Vaccines in Malawi. UCLA. 2019. ProQuest ID: Clair_ucla_0031N_17983. Merritt ID: ark:/13030/m5v74hns.. https://escholarship.org/uc/item/5bj053qp (Accessed Dec. 17, 2020).

40. Msyamboza, KP. Mwagomba, BM. Valle M. Chiumia, H. and Phiri, T. Implementation of a human papillomavirus vaccination demonstration project in Malawi: successes and challenges. BMC Public Health, 17:599 DOI 10.1186/s12889-017-4526-y

41. Magombo-Mana, K. Malawi launches HPV vaccine campaign: Minister Muluzi says cervical cancer leading cause of women's deaths. . 2019, January 12. https://www.nyasatimes.com/malawi-launches-hpv-vaccine-campaign-minister-muluzi- sayscervical-cancer-leading-cause-of-womens-deaths/ (Accessed Dec. 8, 2020).

42. Malawi Ministry of Health (MOH), HPV Vaccine demonstration project evaluation in Malawi. Technical report, May 2014.

43. Malawi Ministry of Health (MOH). Malawi National Routine Immunization data 2019, unpublished, January 2020.

44. Betsch C, Böhm R, Chapman GB. Using behavioral insights to increase vaccination policy effectiveness. Policy Insights, Behav Brain Sci. 2015; 2: 61-73.

45. MacDonald, NE. Vaccine hesitancy: Definition, scope and determinants". SAGE Working Group on Vaccine Hesitancy. 2015; 33(34):4161-4.

46. World Health Organization. Immunization. Improving vaccination demand and addressing hesitancy, World Health Organization, Geneva, 2019.

47. Strategic Advisory Group of Experts (SAGE) on Vaccination Hesitancy Report. World Health Organisation (WHO). $2014 ; 7,18$ https://www.who.int/immunization/sage/meetings/2014/october/SAGE_working_group_revised_report_vaccine_hesitancy.pdf (Accessed April 3, 2021).

48. Dube E, Laberge C, Guay M, Bramadat P, Roy R, Bettinger J. Vaccine hesitancy: An overview. Human vaccines \& immunotherapeutics 2013;9(8):1763-73. doi:10.4161/hv.24657.

49. Betsch C, Schmid P, Heinemeier D, Korn L, Holtmann C, Bö hm R. "Beyond confidence: Development of a measure assessing the 5C psychological antecedents of vaccination". PLOS ONE, 2018; 13(12): e0208601. https://doi.org/10.1371/journal.pone.0208601

50. Larson HJ, de Figueiredo A, Xiahong Z, Schulz WS, Verger P, Johnston IG, et al. "The State of Vaccine Confidence 2016: Global Insights Through a 67-Country Survey”. EBioMedicine. 2016; 12: 295-301. DOI: 1016/j.ebiom.2016.08.042 
51. Cooper, S., Betsch, C., Sambala, EZ., Mchiza, N. \& Wiysonge, CS. Vaccine hesitancy - a potential threat to the achievements of vaccination programmes in Africa, Human Vaccines \& Immunotherapeutics, 2018; 14:10, 2355-2357, DOI:

10.1080/21645515.2018.1460987.

52. WHO-UNICEF Joint Reporting Form (JRF), Malawi Data, 2018.

53. Lane, Sarah. MacDonald, NE. Marti, M. Dumolard, L. Vaccine hesitancy around the globe: Analysis of three years of WHO/UNICEF Joint Reporting Form data-2015-2017, Vaccine, 2018; 36 (26), 3861-3867.

54. Masters N. B., Tefera Y. A., Wagner A. L., Boulton M. L. Vaccine hesitancy among caregivers and association with childhood vaccination timeliness in Addis Ababa, Ethiopia, Human Vaccines and Immunotherapeutics, 2018; 14(10):2340-2347. doi: 10.1080/21645515.2018.1480242.

55. Okeibunor JC, Ogbuanu I, Blanche A, Chiwaya K, Chirwa G, Machekanyanga Z, Mihigo R, Zawaira F. Towards a Strategy for Reducing Missed Opportunities for Vaccination in Malawi: Implications of a Qualitative Health Facility Assessment. Immunol Sci. 2018; Suppl(7):46-54.

56. Ogbuanu IU, Li AJ, Anya B-pM, Tamadji M, Chirwa G, Chiwaya KW, et al. Can vaccination coverage be improved by reducing missed opportunities for vaccination? Findings from assessments in Chad and Malawi using the new WHO methodology. PLOS ONE, 2019; 14(1): e0210648. https://doi.org/10.1371/journal.pone.0210648

57. Vermandere, Heleen, Naanyu, Violet., Degomme, Olivier \& Michielsen, Kristien, Implementation of an HPV vaccination program in Eldoret, Kenya: results from a qualitative assessment by key stakeholders, BMC Public Health, 2015; 15, 875.

58. Vermandere, Heleen. Introduction of HPV vaccination in Kenya. Afrika Focus. 2016; 10.21825/af.v29i2.4851.

59. Remes P, Selestine V, Changalucha J, Ross DA, Wight D, de Sanjosé S, et al. A qualitative study of HPV vaccine acceptability among health workers, teachers, parents, female pupils, and religious leaders in northwest Tanzania. 2012; 3;30(36):5363-7

60. Wiyeh, AB., Cooper, S., Nnaji, CA., Wiysonge, CS. Vaccine hesitancy 'outbreaks': using epidemiological modeling of the spread of ideas to understand the effects of vaccine related events on vaccine hesitancy. Expert Review of Vaccines. 2018;17(12).

61. Karafillakis E, Simas C, Jarrett C, Verger P, Peretti-Watel P, Dib F, et al. HPV vaccination in a context of public mistrust and uncertainty: a systematic literature review of determinants of HPV vaccine hesitancy in Europe. Hum Vaccin Immunother. 2019: 11;21645515.2018.1564436.

62. Costa, Janaína Calu., Weber, Ann M., Darmstadt, Gary L., Abdalla, Safa and Victora, Cesar G. Religious affiliation and immunization coverage in 15 countries in Sub-Saharan Africa, Vaccine, 2019; 38 (5), 1160-1169, https://doi.org/10.1016/j.vaccine.2019.11.024.

63. Ruijs, W.L., Hautvast, J.L., Kerrar, S. et al. The role of religious leaders in promoting acceptance of vaccination within a minority group: a qualitative study. BMC Public Health, 2013; 13, 511. https://doi.org/10.1186/1471-2458-13-511

64. Fournet, N., Mollema, L., Ruijs, WL., Harmsen, IA., Keck, F., Durand, JY., Cunha, MP., Wamsiedel, M., Reis, R., French, J., Smit, EG., Kitching, A., van Steenbergen, JE. Under-vaccinated groups in Europe and their beliefs, attitudes and reasons for nonvaccination; two systematic reviews. BMC Public Health. 2018; 18(1):196. doi: 10.1186/s12889-018-5103-8. PMID: 29378545; PMCID: PMC5789742.

65. Jegede AS: What led to the Nigerian boycott of the polio vaccination campaign? PLoS Med. 2007, 4: e7310.1371/journal.pmed.0040073.

66. Bangura, J.B., Xiao, S., Qiu, D.et al. Barriers to childhood immunization in sub-Saharan Africa: A systematic review. BMC Public Health, 2020; 20, https://doi.org/10.1186/s12889-020-09169-4

67. Oku, A., Oyo-Ita, A., Glenton, C. et al.Factors affecting the implementation of childhood vaccination communication strategies in Nigeria: a qualitative study. BMC Public Health, 2017; 17, https://doi.org/10.1186/s12889-017-4020-6

68. Wiysonge, CS. Vaccine Hesitancy, an Escalating Danger in Africa. Think Global Health. 2019, Dec 17. https://www.thinkglobalhealth.org/article/vaccine-hesitancy-escalating-danger-africa (Accessed Dec. 2, 2020).

69. Bellhouse, D. Systematic sampling methods. Wiley Online Library: Encyclopedia of Biostatistics. 2005. http://onlinelibrary.wiley.com/doi/10.1002/0470011815.b2a16077/abstract.

70. Lane, Sarah. MacDonald, NE. Marti, M. Dumolard, L. Vaccine hesitancy around the globe: Analysis of three years of WHO/UNICEF Joint Reporting Form data-2015-2017, Vaccine, 2018; 36 (26), 3861-3867 
71. Bonem, E. M. Ellsworth, P. C. Gonzalez, Richard. Age Differences in Risk: Perceptions, Intentions and Domains. Journal of Behavioural decision Making, 2015; 28 (4), 317-330. https://doi.org/10.1002/bdm.1848. (Accessed Dec. 5, 2020).

72. Renner, B., Knoll, N., \& Schwarzer, R. Age and body make a difference in optimistic health beliefs and nutrition behaviors. International Journal of Behavioral Medicine, 2000; 7(2), 143159. https://doi.org/10.1207/S15327558IJBM0702_4

73. Rolison, JJ., Hanoch, Y., Wood, S., Liu, P., Risk-Taking Differences Across the Adult Life Span: A Question of Age and Domain, The Journals of Gerontology: 2014; 69 (6), 870-880, https://doi.org/10.1093/geronb/gbt081

74. Arnett, J. Reckless behavior in adolescence: A developmental perspective. Developmental Review, 1992; 12(4), 339-373

75. Lapsley DK and Hill PL. Subjective invulnerability, optimism bias and adjustment in emerging adulthood. J Youth Adolesc. 2010; 39: 847-857. https://doi.org/10.1007/s10964-009-9409-9 PMID: 20596815

76. Madhi, Shabir A. \& Rees, Helen. Special focus on challenges and opportunities for the development and use of vaccines in Africa, Human Vaccines \& Immunotherapeutics,2018; 14:10,2335-2339, DOI: 1080/21645515.2018.1522921

77. Matta, P., El Mouallem, Rayane., Akel, Marwan., Hallit, Souheil and Khalife, Marie-Claude Fadous. Parents' knowledge, attitude and practice towards children's vaccination in Lebanon: role of the parent-physician communication. BMC Public Health, 2020; 20: 1439. doi: 10.1186/s12889-020-09526-3

78. Sambala EZ, Uthman OA, Adamu AA, Ndwandwe D, Wiyeh AB, Olukade T, Bishwajit G, Yaya S, OkwoBele JM, Wiysonge CS. Inequality in missed opportunities for vaccination in sub-Saharan Africa? Compositional and structural characteristics. Hum Vaccin Immunother.2018:1-8. doi:10.1080/21645515.2018.1460985.

79. Mora, T., \& Trapero-Bertran, M. The influence of education on the access to childhood immunization: the case of Spain.BMC public health, 2018; 18(1), 893. https://doi.org/10.1186/s12889-018-5810-1

80. Keneally, Meghan. Parents who don't vaccinate kids tend to be affluent, better educated, experts say. ABC News. 2019, Jan. 29. https://abcnews.go.com/Health/parents-vaccinate-kids-tend-affluent-educated-experts/story?id=60674519 (Accessed Nov. 10, 2020).

81. Zhao, Laijun., Wang, Jiajia., Huang, Rongbing. Immunization against the Spread of Rumors in Homogenous Networks. PLOS ONE, 2015; 10(5): e0124978. https://doi.org/10.1371/journal.pone.0124978

82. Burki, Talha. Vaccine misinformation and social media. The Lancet Digital Health, 2019; 1 (6), E258-E259. https://www.thelancet.com/journals/landig/article/PIIS2589-7500(19)30136-0/fulltext

83. European Centre for Disease Prevention and Control (ECDC). Systematic scoping review on social media monitoring methods and interventions relating to vaccine hesitancy. Stockholm: ECDC, 2020.

84. Steffens, M.S., Dunn, A.G., Wiley, K.E. et al. How organisations promoting vaccination respond to misinformation on social media: a qualitative investigation. BMC Public Health,2019; 19, https://doi.org/10.1186/s12889-019-7659-3

85. Wilson SL, Wiysonge C. Social media and vaccine hesitancy. BMJ Global Health 2020; 5:e004206. doi:10.1136/ bmjgh-2020004206

\section{Supplementary Files}

This is a list of supplementary files associated with this preprint. Click to download.

- Questionnaire.pdf 